\title{
Genetic basis of unstable expression of high gamma-tocopherol content in sunflower seeds
}

\author{
María J García-Moreno, José M Fernández-Martínez, Leonardo Velasco and Begoña Pérez-Vich*
}

\begin{abstract}
Background: Tocopherols are natural antioxidants with both in vivo (vitamin E) and in vitro activity. Sunflower seeds contain predominantly alpha-tocopherol ( $>90 \%$ of total tocopherols), with maximum vitamin E effect but lower in vitro antioxidant action than other tocopherol forms such as gamma-tocopherol. Sunflower germplasm with stable high levels of gamma-tocopherol $(>85 \%)$ has been developed. The trait is controlled by recessive alleles at a single locus Tph2 underlying a gamma-tocopherol methyltransferase (gamma-TMT). Additionally, unstable expression of increased gamma-tocopherol content in the range from 5 to $85 \%$ has been reported. The objective of this research was to determine the genetic basis of unstable expression of high gamma-tocopherol content in sunflower seeds.

Results: Male sterile plants of nuclear male sterile line nmsT2100, with stable high gamma-tocopherol content, were crossed with plants of line IAST-1, with stable high gamma-tocopherol content but derived from a population that exhibited unstable expression of the trait. $F_{2}$ seeds showed continuous segregation for gamma-tocopherol content from 1.0 to 99.7\%. Gamma-tocopherol content in $F_{2}$ plants (average of 24 individual $F_{3}$ seeds) segregated from 59.4 to $99.4 \%$. A genetic linkage map comprising 17 linkage groups (LGs) was constructed from this population using 109 SSR and 20 INDEL marker loci, including INDEL markers for tocopherol biosynthesis genes. QTL analysis revealed a major QTL on LG 8 that corresponded to the gamma-TMT Tph2 locus, which suggested that high gamma-tocopherol lines nmsT2100 and IAST-1 possess different alleles at this locus. Modifying genes were identified at LGs 1, 9, 14 and 16, corresponding in most cases with gamma-TMT duplicated loci.

Conclusions: Unstable expression of high gamma-tocopherol content is produced by the effect of modifying genes on tph $2^{a}$ allele at the gamma-TMT Tph2 gene. This allele is present in line IAST-1 and is different to allele tph2 present in line nmsT2100, which is not affected by modifying genes. No sequence differences at the gammaTMT gene were found associated to allelic unstability. Our results suggested that modifying genes are mostly epistatically interacting gamma-TMT duplicated loci.
\end{abstract}

\section{Background}

Tocopherols are the main antioxidants present in seed oils. They form a family of four fat-soluble compounds with vitamin $\mathrm{E}$ activity named alpha-, beta-, gamma-, and delta tocopherol. Tocopherols contain a 6-chromanol ring structure methylated to varying degrees at positions 5, 7, and 8 , and an isoprenoid-derived C16 saturated side chain at position 2 . The four tocopherols differ by the number and positions of the methyl groups on the 6chromanol ring. Alpha-tocopherol is trimethylated,

\footnotetext{
* Correspondence: bperez@ias.csic.es

Instituto de Agricultura Sostenible (IAS-CSIC), Alameda del Obispo s/n, 14004, Córdoba, Spain
}

beta- and gamma-tocopherol are dimethylated, and deltatocopherol is monomethylated [1].

Tocopherols are important antioxidants operating both in vivo, protecting cells from oxidative damage, as well as in vitro, protecting oils and oil-based products from oxidation [2]. The relative in vivo and in vitro antioxidant properties of the specific tocopherols is determined by their chemical structure. The relative biological activity of the tocopherols is estimated as $100 \%$ for alpha-tocopherol, 15 to $27 \%$ for beta-tocopherol, 3 to $20 \%$ for gammatocopherol, and 0.3 to $2 \%$ for delta-tocopherol [3]. However, there is a widespread confusion concerning their relative potency in vitro [4], though it is generally accepted that alpha-tocopherol shows better antioxidant activity in 
fats and oils at lower concentrations, but at higher concentrations gamma-tocopherol is a more active antioxidant [5]. Studies conducted in sunflower seed oil, in which alpha-tocopherol accounts for more than $>90 \%$ of the total tocopherols, concluded that substitution of alphatocopherol by gamma-tocopherol has a positive impact on the stability of the oil [6-9].

Four sunflower germplasms named LG-17, T2100, IAST-1, and IAST-540 in which alpha-tocopherol in the seeds was almost completely replaced by gammatocopherol have been developed [6,10,11]. Gammatocopherol in the novel germplasm accounts for more than $85 \%$ of the total seed tocopherols, compared with more than 90\% alpha-tocopherol in conventional sunflower seeds. The increased gamma-tocopherol levels are produced by recessive alleles at the Tph2 locus $[6,12,13]$, which encodes a gamma-tocopherol methyltransferase (gamma-TMT) enzyme [14]. This enzyme catalyzes the methylation of delta- and gamma-tocopherol to yield beta- and alpha-tocopherol, respectively [15]. GammaTMT mutation in sunflower disrupts the synthesis of alpha-tocopherol and causes the accumulation of gamma-tocopherol [14]. In a detailed sequence analysis of the gamma-TMT gene in sunflower, Hass et al. [14] identified two gamma-TMT paralogs (gamma-TMT-1 and gamma-TMT-2) and five different haplotypes (haplotypes 4 and 5 corresponding to paralogs 1 and 2, respectively). Both gamma-TMT paralogs 1 and 2 cosegregated with Tph2 and were mapped to linkage group (LG) 8 of the sunflower linkage map. Even though the Tph 2 mutation reduced or disrupted the expression of the two paralogs in developing sunflower seeds, none of the DNA polymorphisms found within the gammaTMT Tph2 gene were associated with the high gammatocopherol phenotype [14]. The authors suggested that the Tph 2 mutation must be very tightly linked to the gamma-TMT locus on LG 8 and may disrupt regulatory sequences.

Phenotypic studies of Demurin et al. [16] and GarcíaMoreno et al. [13] concluded that the four high gammatocopherol lines LG-17, T2100, IAST-1, and IAST-540 possess the same allele at Tph2, as no transgressive segregations were observed in crosses involving the four lines. Lines LG-17, T2100 and IAST-540 were isolated from germplasm or $M_{1}$ mutant plants that segregated for high alpha-tocopherol (>90\%) and high gammatocopherol $(>85 \%)$, with no intermediate levels of both tocopherol forms being observed [6,10-12]. Conversely, IAST-1 was isolated from an $\mathrm{M}_{2}$ plant that exhibited large variation for alpha- and gamma-tocopherol levels, with gamma-tocopherol content in $\mathrm{M}_{3}$ seeds showing a continuous variation from zero to $84.6 \%$ and $\mathrm{M}_{4}$ seeds from selected $\mathrm{M}_{3}$ plants showing a variation from 60.4 to $97.4 \%$ gamma-tocopherol, which was uniformly high
(>90\%) in $M_{5}$ seeds from selected $M_{4}$ plants [11]. García-Moreno et al. [17] suggested that the intermediate gamma-tocopherol levels observed during the isolation of IAST-1 might be produced by the presence of modifying genes that determined unstable expression of mutated alleles at Tph2 locus. Modifying genes have been found to influence important traits in sunflower such as high oleic acid content [18] or broomrape resistance [19].

In this study, the genetic analysis of a population that showed segregation from low to high gamma-tocopherol values obtained from the cross between the two high gamma-tocopherol lines IAST-1 and nmsT2100 has been carried out. In the course of this analysis, we identified two different alleles at the gamma-TMT Tph2 locus at LG 8. The allele tph2 was present in line nmsT2100, whereas the allele $t p h 2^{a}$ was identified in line IAST-1. Additionally, we found four modifying genes at LGs $1,9,14$ and 16 that in most cases corresponded to duplicated gamma-TMT loci. Modifying genes influenced the expression of $t p h 2^{a}$ alleles, but did not affect tph2 alleles.

\section{Results}

\section{Phenotypic segregations}

Seeds of sunflower lines nmsT2100 (fertile plants) and IAST-1 showed uniformly high gamma-tocopherol content, from 91.2 to $99.8 \%$ in nmsT2100 and from 92.3 to 99.4\% in IAST-1. $F_{1}$ seeds from the cross between nmsT2100 and IAST-1 had also high gamma-tocopherol content, from 92.6 to $97.2 \%$. However, large segregation for gamma-tocopherol content was observed in $\mathrm{F}_{2}$ seeds from some $\mathrm{F}_{1}$ plants. Particularly, the analysis of $192 \mathrm{~F}_{2}$ seeds from an $F_{1}$ plant derived from an $F_{1}$ seed with 95.5\% gamma-tocopherol revealed a continuous large segregation for the trait, from 1.0 to $99.7 \%$, with no discrete phenotypic classes being observed and a nonnormal distribution $(\mathrm{p}<0.0001$; Kolmogorov-Smirnov test) (Figure 1). Variation for gamma-tocopherol content was smaller at the $\mathrm{F}_{2}$ plant generation (average of 24 individual $\mathrm{F}_{3}$ seeds), which segregated from 59.4 to $99.4 \%$ (Figure 2). However, examination of variation of individual $F_{3}$ seeds within each $F_{2}$ plant showed that minimum gamma-tocopherol content in individual $\mathrm{F}_{3}$ seeds from the different $\mathrm{F}_{3}$ families ranged from 0.0 to $98.4 \%$, whereas maximum gamma-tocopherol content was in all cases above $94 \%$. No discrete classes could be distinguished for minimum gamma-tocopherol content in the $\mathrm{F}_{2: 3}$ population (data not shown).

\section{Map construction and candidate gene mapping}

One hundred and sixty three out of 437 (37.3\%) simple sequence repeat (SSR) and insertion-deletion (INDEL) markers were polymorphic in the screening of the high 

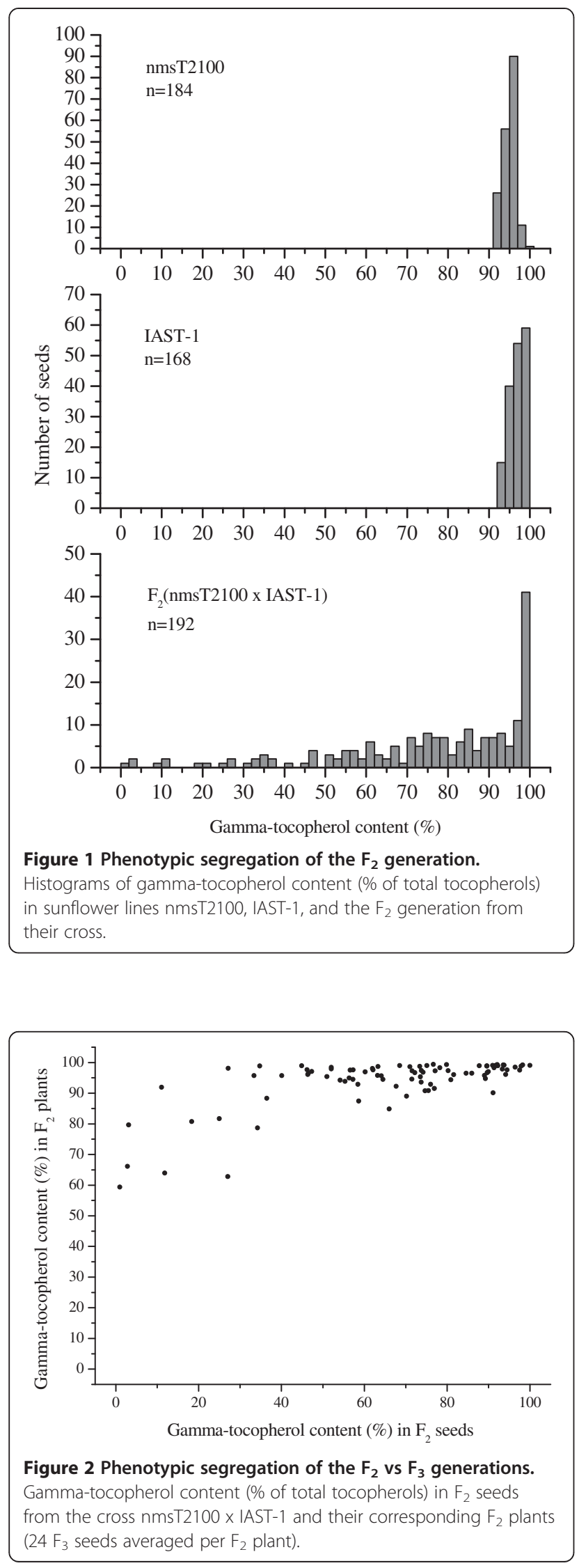

gamma-tocopherol lines nmsT2100 and IAST-1 and four randomly selected $F_{2}$ individuals from their cross. A set of 128 high quality, evenly spaced, and preferably codominant markers were genotyped in the $\mathrm{F}_{2}$ population. Final linkage map for QTL analyses comprised 129 marker loci (109 SSR and 20 INDEL) grouped on 17 linkage groups (Table 1). The total genetic distance covered by these marker loci was $792.4 \mathrm{cM}$, with an average marker interval of $7.0 \mathrm{cM}$ (Table 1). Linkage groups contained between 3 and 16 marker loci, with $99.6 \%$ of the mapped genome being within $20 \mathrm{cM}$ to the nearest marker. No marker loci had significantly distorted segregation ratios $(\mathrm{P}<0.001)$.

INDEL markers for the gamma-TMT gene described by Hass et al. [14] were not polymorphic between nmsT2100 and IAST-1 or showed polymorphism in secondary loci. Therefore, new primer combinations based on the full-lenght sequence of the gamma-TMT gene in nmsT2100 and IAST-1 (see below) were designed and tested. A primer combination (gamma-TMT-F1/F2/R24) based on the forward primers F1 and F2 placed upstream and dowstream, respectively, of the T39 transcription initiation site [14], and a reverse primer R24 complementary to the DNA sequence in exon 2

Table 1 nmsT2100 x IAST-1 linkage map coverage

\begin{tabular}{llllll}
\hline & \multicolumn{5}{c}{$\begin{array}{l}\text { LG coverage } \\
\text { Coverage }\end{array}$} \\
\hline LG $^{\mathbf{2}}$ & SSR & INDEL & Mean & Largest interval & Total \\
\hline 1 & 13 & 3 & 4.3 & 19.3 & 64.0 \\
2 & 6 & 0 & 4.4 & 8.2 & 22.1 \\
3 & 6 & 1 & 16.6 & 27.8 & 99.7 \\
4 & 5 & 1 & 4.2 & 8.1 & 20.9 \\
5 & 5 & 1 & 5.5 & 15.5 & 27.6 \\
6 & 5 & 2 & 3.6 & 13.4 & 21.5 \\
7 & 7 & 1 & 10.7 & 43.4 & 74.9 \\
8 & 8 & 3 & 4.5 & 14.9 & 44.6 \\
9 & 6 & 0 & 6.6 & 22.8 & 32.9 \\
10 & 7 & 4 & 10.1 & 29.8 & 100.9 \\
11 & 3 & 0 & 6.6 & 8.9 & 13.2 \\
12 & 4 & 2 & 4.3 & 7.5 & 21.3 \\
13 & 7 & 0 & 8.3 & 31.2 & 50.0 \\
14 & 7 & 1 & 4.6 & 10.2 & 32.4 \\
15 & 6 & 0 & 10.1 & 36.5 & 50.5 \\
16 & 8 & 1 & 9.6 & 32.1 & 76.8 \\
17 & 6 & & 7.8 & 17.1 & 39.1 \\
Total & 109 & 20 & & & 792.4 \\
\hline & & & &
\end{tabular}

${ }^{1} \mathrm{LG}=$ Linkage group.

${ }^{2} \mathrm{SSR}=$ Number of SSR loci; INDEL = Number of INDEL loci. Genome coverage offered by the marker set used for QTL analysis in the nmsT2100 x IAST-1 population. Distances are expressed in Haldane cM. 


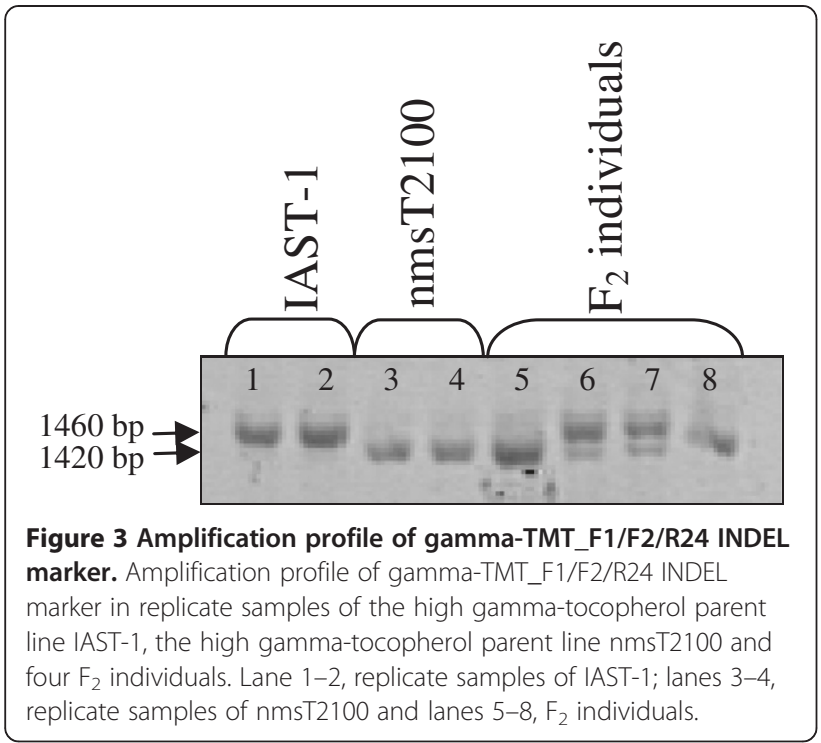

amplified a primary gamma-TMT locus that was polymorphic between IAST-1 and nmsT2100 (Figure 3). The polymorphic band was about 1460 bp in IAST-1 and $1420 \mathrm{bp}$ in nmsT2100 (Figure 3). When screened against the individuals from the $\mathrm{F}_{2}$ population, the gamma-TMTF1/F2/R24 locus was co-dominantly mapped to LG 8 at the previously described position of the gamma-TMT Tph2 gene [14]. Other INDEL markers for the gammaTMT gene were also tested. The F9/R24 gamma-TMT INDEL marker showed three loci in the nmsT2100 and IAST-1 parental lines (Figure 4). A primary locus (gamma-TMT-F9/R24a) corresponding to a band of about 1200 bp was not polymorphic with this primer combination (Figure 4). This locus, however, was demostrated to co-segregate with Tph2 and was co-dominantly mapped to LG 8 in the population CAS-12 x IAST-540, also segregating for gamma-tocopherol content [13]. A second locus (gamma-TMT-F9/R24b) that showed a dominant polymorphism, with a band of about 750 bp present in nmsT2100 and absent in IAST-1, was mapped to LG 16 (Figure 4). The locus was also mapped to LG 16 in the CAS-12 x IAST-540 population. Finally, a third locus (gamma-TMT-F9/R24c) that also showed a dominant polymorphism, with a band of $375 \mathrm{bp}$ absent in nmsT2100 and present in IAST-1, was mapped to LG 1 (Figure 4). In addition to the gamma-TMT-F9/R24a, b and c loci, a fourth gamma-TMT locus was identified in populations other than nmsT2100 x IAST-1. This locus was named gamma-TMT-F9/R24d and was mapped to LG 14 in populations CAS-12 x IAST-540 [13] and IAST-413 x HA-89 (Del Moral L, unpublished data) (Figure 4). Finally, the locus MT-2 of the 2-methyl-6-phytyl-1,4-benzoquinone/2-methyl-6-solanyl-1,4-benzoquinone methyltransferase (MPBQ/MSBQ-MT) gene was mapped to LG 4 using the F24/R25 INDEL marker [20]. None of the other markers for tocopherol biosynthesis genes tested were polymorphic between nmsT2100 and IAST-1.

\section{QTL analyses}

One-factor analysis of variance revealed that the gamma-TMT-F1/F2/R24 locus on LG 8 was underlying a major QTL affecting gamma-tocopherol content. This locus had a significant effect on gamma-tocopherol content of the $\mathrm{F}_{2}$ and the $\mathrm{F}_{3}$ generations (Table 2) and corresponded to the gamma-TMT Tph2 gene also mapped to the same position on LG 8 by Hass et al. [14]. The mean and the standard deviation for gamma-tocopherol

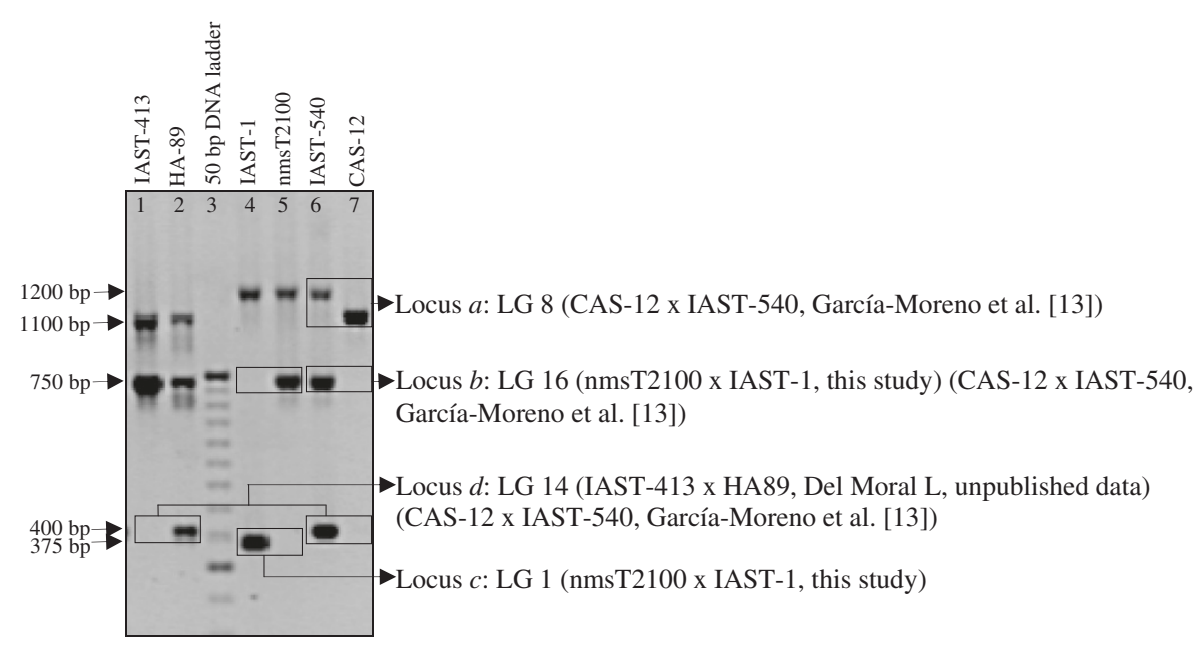

Figure 4 Amplification profile of gamma-TMT_F9/R24 INDEL marker. Amplification profile of gamma-TMT_F9/R24 INDEL marker in single samples of the high gamma-tocopherol IAST-1, nmsT2100, IAST-540 lines and the standard gamma-tocopherol IAST-413, HA-89 and CAS-12 lines. Lane 1, IAST-413; lane 2, HA-89; lane 3, 50 bp DNA ladder; lane 4, IAST-1; lane 5, nmsT2100; lane 6, IAST-540 and lane 7, CAS-12. Polymorphic loci are highlighted by boxes, and the linkage group and the population in which they have been mapped are shown at the right of the figure. 
Table 2 Effect of gamma-TMT-F1/F2/R24 on seed gamma-tocopherol content

\begin{tabular}{|c|c|c|c|c|c|c|c|c|}
\hline \multirow[t]{2}{*}{$\begin{array}{l}\text { Seed } \\
\text { generation }\end{array}$} & \multicolumn{3}{|c|}{$\begin{array}{l}\text { No. individuals within } \\
\text { each marker class }\end{array}$} & \multicolumn{3}{|c|}{$\begin{array}{l}\text { Mean } \pm \text { SD for gamma-tocopherol content } \\
(\% \text { total tocopherols)within each marker class }\end{array}$} & \multicolumn{2}{|c|}{ ANOVA analysis } \\
\hline & A & $\mathrm{H}$ & B & $\mathrm{A}(\mathrm{IAST}-1)$ & $\mathrm{H}$ & B(nmsT2100) & $F$ & P \\
\hline $\mathrm{F}_{2}$ & 32 & 73 & 27 & $52.3 a \pm 29.6$ & $72.2 b \pm 19.1$ & $98.9 c \pm 0.8$ & 38.6 & $<0.001$ \\
\hline $\mathrm{F}_{3}$ & 26 & 58 & 3 & $86.8 a \pm 11.8$ & $96.9 b \pm 2.6$ & $98.6 b \pm 0.9$ & 20.4 & $<0.001$ \\
\hline
\end{tabular}

Mean values within rows followed by the same letter are not significantly different at 0.05 level of probability (Duncan's multiple range test). Association between the gamma-TMT-F1/F2/R24 locus on LG 8 and gamma-tocopherol content (\% of the total tocopherols) determined by variance analysis in the nmsT2100 x IAST-1 population. Mean gamma-tocopherol \pm standard deviation (SD) are presented in different genotypic classes: $A=$ homozygous with respect to the allele derived from IAST-1, B = homozygous with respect to the allele derived from nmsT2100, $\mathrm{H}=$ heterozygous.

content in the $\mathrm{F}_{2}$ seed generation of plants homozygous for the IAST-1 allele were $52.3 \pm 29.6 \%$, whereas those for plants homozygous for the nmsT2100 allele were $98.9 \pm 0.8 \%$ (Table 2). These results suggested the existence to two different alleles at the gamma-TMT Tph2 locus in lines nmsT2100 and IAST-1. The allele at IAST-1 was unstable, producing in homozygous condition a broad distribution ranging from 0.97 to $89.02 \%$ in the $F_{2}$ seed generation, whereas the allele at nmsT2100 was highly stable and resulted in gamma-tocopherol values above $97 \%$. Similar results were observed in the $\mathrm{F}_{3}$ seed generation (Table 2). It is interesting to note that only three $\mathrm{F}_{2}$ plants homozygous for the nmsT2100 allele produced sufficient number of $\mathrm{F}_{3}$ seeds for tocopherol analyses, compared to $26 \mathrm{~F}_{2}$ plants homozygous for the IAST-1 allele (Table 2). This was probably caused by a close linkage between Tph2 and Ms11 loci at LG 8, the latter responsible for male sterility in the nuclear male

Table 3 QTL affecting gamma-tocopherol content in the nmsT2100 x IAST-1 population

\begin{tabular}{|c|c|c|c|c|c|c|c|c|c|}
\hline \multirow[b]{2}{*}{ Generation } & \multirow[b]{2}{*}{ QTL } & \multirow[b]{2}{*}{$\mathrm{LG}^{1}$} & \multirow[b]{2}{*}{ Pos. $^{2}$} & \multirow[b]{2}{*}{ Supp int. ${ }^{3}$} & \multirow[b]{2}{*}{ Marker interval $^{4}$} & \multirow[b]{2}{*}{ LOD } & \multirow[b]{2}{*}{$R^{2}(\%)$} & \multicolumn{2}{|c|}{ Significant gene effects } \\
\hline & & & & & & & & $a$ & $d$ \\
\hline \multirow[t]{10}{*}{$\overline{F_{2}}$} & GamT1.1 & 1 & 44 & $29-63$ & ORS552 to Gamma_TMT_F9/R24C & 2.4 & 7.8 & & $-2.96^{*}$ \\
\hline & Tph2_Gamma-TMT & 8 & 12 & $7-15$ & ZVG34 to Gamma_TMT_F1/F2/R24 & 15.0 & 41.2 & $20.9^{* *}$ & \\
\hline & GamT9.1 & 9 & 32 & $19-32$ & ORS887 to ORS176 & $2.3^{6}$ & 7.5 & $-7.7^{* *}$ & \\
\hline & GamT14.1 & 14 & 16 & $6-17$ & ORS185 to ORS307 & 4.1 & 13.1 & $-11.5^{* *}$ & \\
\hline & GamT16.1 & 16 & 13 & $11-21$ & Gamma_TMT_F9/R24b to ORS700 & 3.4 & 10.9 & & \\
\hline & & & & & Total & 17.2 & 44.4 & & \\
\hline & & & & & addTph2_Gamma-TMT*addGamT14.1 & & & $9.9^{*}$ & \\
\hline & & & & & addTph2_Gamma-TMT*addGamT16.1 & & & $14.0^{* *}$ & \\
\hline & & & & & Total epistasis & 21.7 & 52.4 & & \\
\hline & & & & & & & & Signific & ne effects ${ }^{5}$ \\
\hline Generation & QTL & $\mathrm{LG}^{1}$ & Pos. $^{2}$ & Supp int. $^{3}$ & Marker interval $^{4}$ & LOD & $R^{2}(\%)$ & $a$ & $d$ \\
\hline \multirow[t]{9}{*}{$\mathrm{F}_{3}$} & Tph2_Gamma-TMT & 8 & 20 & $16-23$ & Gamma_TMT_F1/F2/R24 to ORS70 & 11.1 & 44.0 & $10.9^{* *}$ & \\
\hline & GamT9.1 & 9 & 31 & $13-32$ & ORS887 to ORS176 & $2.04^{6}$ & 10.1 & $-2.6^{* *}$ & \\
\hline & GamT14.1 & 14 & 16 & $6-27$ & ORS185 to ORS307 & 2.7 & 13.0 & $-3.5^{* *}$ & $2.5^{*}$ \\
\hline & GamT16.1 & 16 & 14 & $13-16$ & ORS700 to ORS757 & 3.3 & 15.9 & $10.6^{* *}$ & $-22.9^{* *}$ \\
\hline & & & & & Total & 18.1 & 61.2 & & \\
\hline & & & & & addTph2_Gamma-TMT*addGamT9.1 & & & $6.8^{* *}$ & \\
\hline & & & & & addTph2_Gamma-TMT*addGamT14.1 & & & $6.7^{* *}$ & \\
\hline & & & & & addTph2_Gamma-TMT*addGamT16.1 & & & $8.5^{* *}$ & \\
\hline & & & & & Total epistasis & 23.8 & 71.3 & & \\
\hline
\end{tabular}

** $=$ significant at the 0.01 and ${ }^{*}=$ significant at the 0.05 probability level.

${ }^{1} \mathrm{LG}=$ Linkage group.

${ }^{2}$ Absolute position from the top of the LG in centiMorgans (cM).

${ }^{3}$ One-LOD support interval in centiMorgans: Refers to the region flanking each QTL peak in which LOD scores decline by one.

${ }^{4}$ Markers flanking the likelihood peak for a putative QTL.

${ }^{5} a=$ additive effect. A positive sign means an increase of the trait value due to nmsT2100 alleles. $d=$ dominant effect. $a$ and $d$ estimates, as well as total $R^{2}$ and

LOD score values were obtained from a simultaneous fit of all putative QTL using multiple regression.

${ }^{6} \mathrm{QTL}$ detected below the LOD threshold. 
sterile (NMS) line P21 [21] from which Ms11 was introgressed to nmsT2100.

Composite interval mapping analyses confirmed the existence of a QTL with a main effect centered on the gamma-TMT-F1/F2/R24 locus on LG 8 (Table 3 and Figure 5). This QTL was named Tph2-gamma-TMT and explained $41.2 \%$ and $44.4 \%$ of the $F_{2}$ and $F_{3}$ gammatocopherol phenotypic variance, respectively (Table 3). A second QTL peak on LG $816 \mathrm{cM}$ apart from Tph2Gamma-TMT was identified only in the QTL analysis of the $\mathrm{F}_{3}$ data (Figure 5). This adjacent peak might be a ghost QTL resulting from the distorted $F_{3}$ data in this region and was not taken into consideration to protect against type I errors in declaring QTLs.

Besides Tph2-Gamma-TMT, four other QTL with a moderate effect on gamma-tocopherol content were
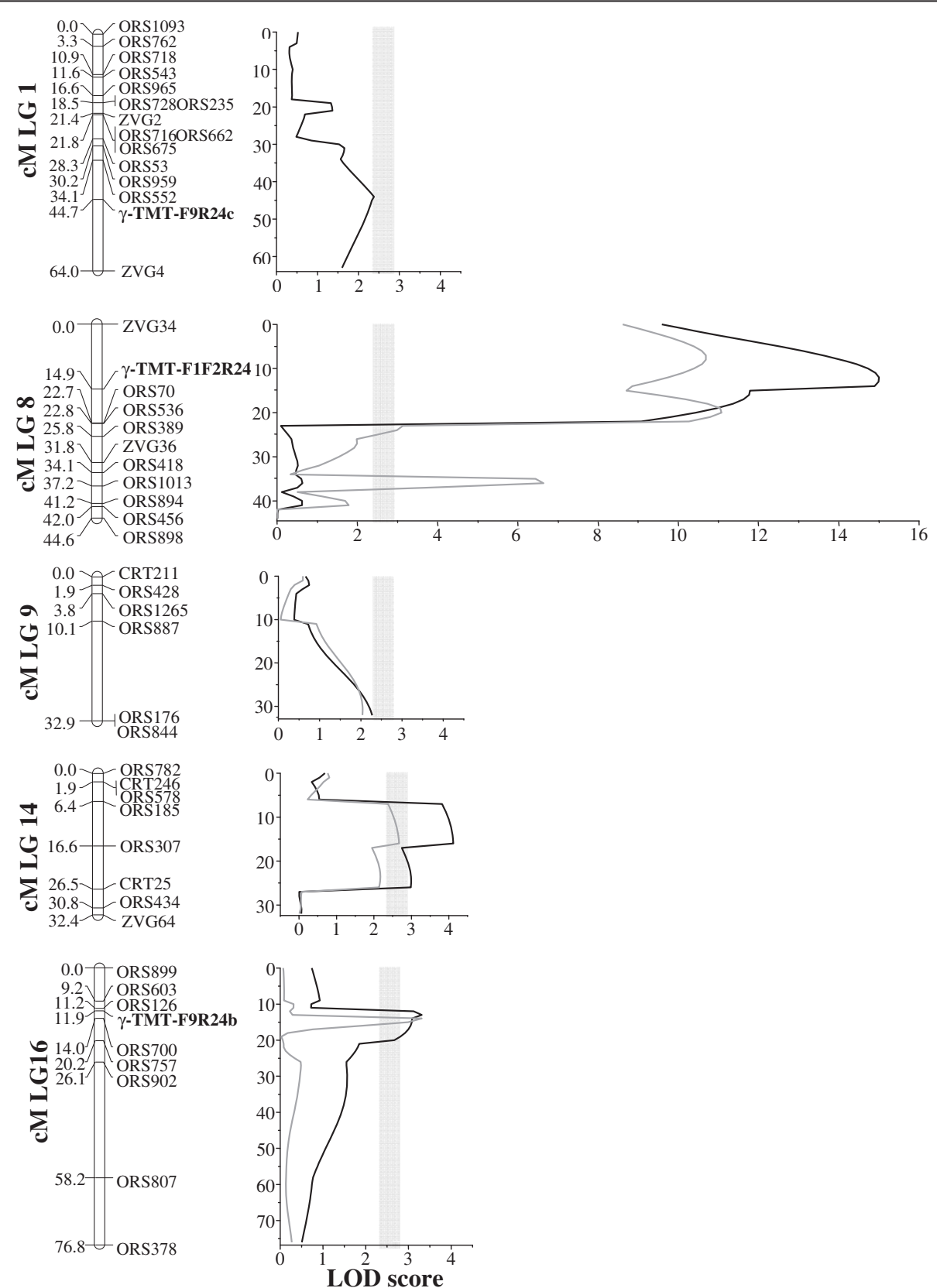

Figure 5 LODs for gamma-tocopherol QTL. Likelihood odds (LODs) for $F_{2}$ (black line) and $F_{3}$ (grey line) gamma-tocopherol QTL on linkage groups (LGs) 1, 8, 9, 14 and 16 in nmsT2100 x IAST-1. Gamma-tocopherol-TMT ( $\gamma$-TMT) loci are highlighted in bold. The thresholds of the LOD score are indicated by grey boxes corresponding to their 0.95 confidence intervals (from 2.48 to 2.76) for gamma-tocopherol content in the $F_{2}$ and the $F_{3}$ generations. 
observed at the $\mathrm{F}_{2}$ level. These QTL were located at LG 1 (GamT1.1), 9 (GamT9.1), 14 (GamT14.1), and 16 (GamT16.1). They individually accounted for 2.3-4.1\% of trait variation (Table 3 and Figure 5). The QTL on LG 9, 14 and 16 were also detected in the $\mathrm{F}_{3}$ analyses at practically identical positions (Table 3 and Figure 5). The model with all the QTL explained $44.4 \%$ and $61.2 \%$ of the phenotypic variance for gamma-tocopherol content in the $\mathrm{F}_{2}$ and the $\mathrm{F}_{3}$ generations, respectively. The QTL peaks of GamT1.1 and GamT16.1 were centered on the gamma-TMT loci mapped at these linkage groups (gamma-TMT-F9/R24c in LG1 and gamma-TMT-F9 /R24b in LG 16) (Figure 5). Additionally, the QTL peak of GamT14.1 was likely to correspond to the gammaTMT-F9/R24d locus mapped to LG 14 in populations CAS-12 x IAST-540 [13] and IAST-413 x HA-89 (Del Moral L, unpublished data), located $1.5 \mathrm{cM}$ from the ZVG64 marker locus at this LG.

Analysis for epistasis in the $F_{2}$ generation through two-way ANOVA revealed significant interactions for gamma-tocopherol content involving the Tph2-gammaTMT marker locus on LG 8 and markers on LG 1, LG 14 and LG 16. The most significant interactions involved the ORS185 marker locus on LG $14 \quad(F=10.5$, $P<0.0001)$, the gamma-TMT_F9R24b locus on LG 16 $(F=4.22, P=0.017)$, and the gamma-TMT_F9R24c locus on LG $1(F=3.12, P=0.048)$, which were associated to the GamT14.1, GamT16.1, and GamT1.1 QTL, respectively. Significant interactions involving the Tph2Gamma-TMT QTL on LG 8, the gamT14.1 on LG 14, and the gamT16.1 QTL on LG 16 were also detected in the composite interval mapping analyses, with the percentage of explained phenotypic variance increasing from $44.4 \%$ to $52.4 \%$ in the $\mathrm{F}_{2}$ and from $61.2 \%$ to $71.3 \%$ in the $\mathrm{F}_{3}$ when epistatic interactions were included in the multiple-locus model (Table 3). Genotypic means for $\mathrm{F}_{2}$ gamma-tocopherol content in allelic combinations of the epistatically interacting marker loci were calculated to detail these interactions. Since ORS185 and gammaTMT_F9R24b marker loci were dominant, genotypic means were computed using close co-dominant markers, ORS578 on LG 14 and ORS126 on LG 16 (Table 4). The results showed that QTL regions on LG 14 and LG 16 only had a significant phenotypic effect when the IAST-1 allele was present at the Tph2-gamma-TMT locus on LG 8 (gamma-TMT-F1/F2/R24). Thus, no significant differences were observed between different genotypes at ORS578 on LG 14 or ORS126 on LG 16 in presence of the nmsT2100 allele at Tph2. However, when the Tph2 locus was homozygous for the IAST-1 allele, the presence of nmsT2100 alleles at ORS578 or ORS126 resulted in a drastic reduction of gamma-tocopherol content as compared to the presence of IAST-1 alleles (Table 4).

\section{Sequence analysis of gamma-TMT loci}

Several loci amplified by the gamma-TMT F9/R24 INDEL marker in IAST-1 and nmsT2100 (Figure 4) were cloned and sequenced. A band of about $1200 \mathrm{bp}$ from the non-polymorphic locus $a$ and a band of about 375 bp from the dominant locus $c$ that mapped to LG 1 were sequenced from IAST-1. A band of about $1200 \mathrm{bp}$ from the non-polymorphic locus $a$ and a band of about 750 bp from the dominant locus $b$ that mapped to LG 16 were sequenced from nmsT2100. The locus $d$ that mapped to LG 14 was sequenced from lines IAST-540 and HA-89.

The locus a fragment isolated from nmsT2100 harboured alleles from both gamma-TMT paralogs, which showed showed $100 \%$ sequence identity to gamma-TMT haplotype 4 (paralog 1) and 5 (paralog 2) from Hass

Table 4 Effect of epistatically interacting marker loci on seed gamma-tocopherol content

\begin{tabular}{|c|c|c|c|c|c|c|c|c|}
\hline \multirow[b]{2}{*}{ Genotype ofy-TMT-F1/F2/R24(LG 8) } & \multirow[b]{2}{*}{ Genotype of ORS578(LG 14) } & \multicolumn{3}{|c|}{$F_{2}$ gamma- $^{1}$} & \multirow[b]{2}{*}{ Genotype of ORS126(LG 16) } & \multicolumn{3}{|c|}{$F_{2}$ gamma- $T^{1}$} \\
\hline & & Mean & SD & $\mathbf{n}$ & & Mean & SD & $\bar{n}$ \\
\hline \multirow[t]{3}{*}{ A (IAST-1) } & B (nmsT2100) & $23.48 a$ & 24.10 & 9 & B (nmsT2100) & $51.68 a b$ & 31.89 & 14 \\
\hline & $\mathrm{H}$ & $64.60 \mathrm{~b}$ & 20.27 & 12 & $\mathrm{H}$ & $44.55 a$ & 27.08 & 14 \\
\hline & A (IAST-1) & $67.48 b$ & 22.69 & 10 & A (IAST-1) & $81.26 \mathrm{~cd}$ & 6.96 & 4 \\
\hline \multirow[t]{3}{*}{$\mathrm{H}$} & $B(n m s T 2100)$ & $71.61 b$ & 18.93 & 18 & B (nmsT2100) & $77.84 \mathrm{C}$ & 16.68 & 22 \\
\hline & $\mathrm{H}$ & $71.00 \mathrm{~b}$ & 19.19 & 42 & $\mathrm{H}$ & $69.17 b c$ & 18.71 & 36 \\
\hline & A (IAST-1) & $76.91 b$ & 19.60 & 13 & A (IAST-1) & $71.23 c$ & 22.38 & 15 \\
\hline \multirow[t]{3}{*}{ B (nmsT2100) } & B (nmsT2100) & $98.92 \mathrm{C}$ & 0.53 & 9 & B (nmsT2100) & $98.59 d$ & 0.97 & 7 \\
\hline & $\mathrm{H}$ & $98.55 c$ & 0.87 & 9 & $\mathrm{H}$ & $98.96 d$ & 0.71 & 16 \\
\hline & A (IAST-1) & $99.15 c$ & 0.86 & 9 & A (IAST-1) & $99.03 d$ & 0.82 & 4 \\
\hline
\end{tabular}

Values within columns followed by the same letter are not significantly different at 0.05 level of probability (Duncan's multiple range test).

${ }^{1}$ Mean and standard deviation (SD) for $F_{2}$ gamma-T content and number of $F_{2}$ individuals per genotypic class (n).

Genotypic means for $F_{2}$ gamma-tocopherol (gamma-T) content (\% of the total tocopherols) in allelic combinations at epistatically interacting marker loci.

$A=$ homozygous with respect to the allele derived from IAST-1, $B=$ homozygous with respect to the allele derived from nmsT2100, $H=$ heterozygous. 
et al. [14]. For IAST-1, the locus $a$ sequence showed $100 \%$ sequence identity to gamma-TMT haplotype 4 . The locus $b$ consensus sequence was 785 bp long and showed a significant homology to gamma-TMT haplotypes 2 and 3 (GenBank accessions nos. DQ229829 and DQ229830, $3 \mathrm{e}^{-58}$ ), with $94 \%$ maximum sequence identity spanning $61 \%$ of the locus $b$ fragment coverage. The consensus sequence for locus $c$ (388 bp long) showed a significant homology to gamma-TMT haplotypes 4 and 5 (GenBank accessions nos. DQ229831 to DQ229834, $4 \mathrm{e}^{-14}$ ), with $86 \%$ maximum sequence identity spanning $19 \%$ of the locus $c$ fragment coverage. The sequences of locus $d$ from IAST-540 (405 bp) and HA-89 (406 bp) were very similar, with a $96 \%$ of sequence identity between them. The consensus sequence obtained from this alignment showed significant homology to gamma-TMT haplotypes 1, 3, 4 and 5 (GenBank accession nos. DQ229828, DQ229830, and DQ229831 and DQ229834; $8 \mathrm{e}^{-18}$ ), with $89 \%$ maximum sequence identity spanning $18 \%$ of the fragment coverage.

Nucleotide sequences from IAST-1 locus $a$, nmsT2100 locus $a$, nmsT2100 locus $b$, IAST- 1 locus $c$, HA-89 locus $d$, and IAST-540 locus $d$ were aligned. The sequence alignment tree (Figure 6) revealed great similarity between loci $a$ and $b$ (85 to $87 \%$ of sequence identity) and between loci $c$ and $d$ (92-93\% of sequence identity). Both groups were more distantly related, with 56 to $58 \%$ of sequence identity between locus $a$ and the cluster of loci $c$ and $d$.

\section{Full-length gamma-TMT genomic DNA sequences}

Agarose gels from PCR products obtained with the F1 and R92 primers flanking the gamma-TMT gene mapped to LG 8 [14] revealed two bands in nmsT2100 and IAST-1 lines that were identified as the two gammaTMT paralogs 1 and 2 described by Hass et al. [14]. Consensus nucleotide sequences for IAST-1 gammaTMT paralog 1 (4126 bp) and paralog 2 (4280 bp) were identical to gamma-TMT haplotype 4 (GenBank accessions nos. DQ229831 and DQ229832) and 5 (GenBank accessions nos. DQ229833 to DQ229834), respectively, from Hass et al. [14] (Additional file 1: Figure S1). Consensus nucleotide sequence for nmsT2100 gamma-TMT paralog 1 (4126 bp) was identical to gamma-TMT haplotype 4 [14] (Additional file 1: Figure S1). Consensus nucleotide sequence for nmsT2100 gamma-TMT paralog 2 (4281 bp) was almost identical to gamma-TMT haplotype 5 [14], with the exception of five SNPs, one in the $5^{\prime}$ UTR ( $\mathrm{G}$ to $\left.\mathrm{A}\right)$, one in intron 1 ( $\mathrm{C}$ to $\mathrm{T}$ ), and three in intron 4 (A to T) (Additional file 1: Figure S1).

\section{Discussion}

The results of this research suggest that high gammatocopherol lines IAST-1 and nmsT2100 possess different mutated alleles at the gamma-TMT Tph2 locus on LG 8. The gamma-TMT catalyses the methylation step from gamma-tocopherol to alpha-tocopherol in sunflower seeds [14]. Mutated tph2 alleles disrupt the activity of gammaTMT, which results in accumulation of gammatocopherol [14]. The allele at nmsT2100 (tph2) is stable and not affected by modifying genes, whereas the allele at IAST-1 $\left(t p h 2^{a}\right)$ is unstable and affected by modifying genes. Putative modifying genes located at LG 1, 9, 14 and 16 were identified in the present research, being the effect of those on LG 1, 14 and 16 highly significant in the epistatic interaction with the Tph2 locus on LG 8. Studies on

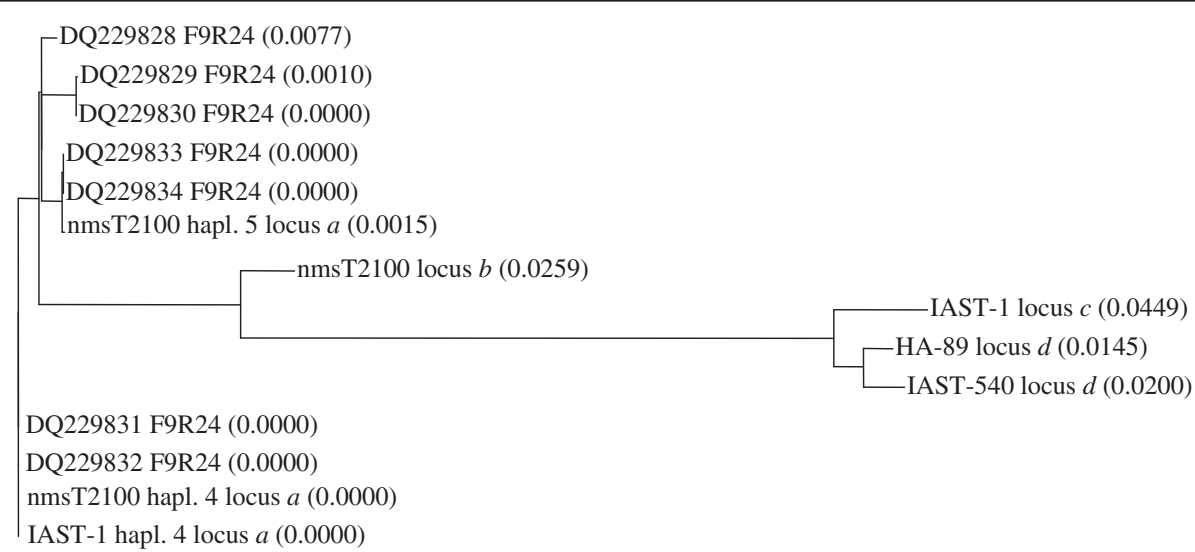

Figure 6 Sequence alignment tree for gamma-TMT loci. Sequence alignment tree obtained by aligning DNA genomic sequences from the different loci amplified with primer combination gamma-TMT F9/R24 and constructed using the AlignX program in the Vector NTI software suite. Calculated distance based on distances between all pairs of sequence values are shown in parenthesis following the molecule name. The locus $a$ fragment isolated from nmsT2100 and IAST-1, the locus $b$ isolated from nmsT2100, the locus $c$ isolated from IAST-1, and the locus $d$ isolated from HA-89 and IAST-540, together with the F9 to R24 region from gamma-TMT haplotypes 1 (DQ DQ229828), 2 (DQ DQ229829), 3 (DQ DQ229830), 4 (DQ DQ229831 and DQ DQ229832), and 5 (DQ DQ229833 and DQ DQ229834) are included. 
expression of Tph 2 mutations in plant tissues other than seeds also pointed to differences between T2100 and IAST-1 lines, as the latter showed lower gammatocopherol content in leaves, roots, and pollen [22].

High gamma-tocopherol lines T2100 and IAST-1 were isolated following different strategies. T2100 derived from an open-pollinated cultivar that showed variation for high gamma-tocopherol content $(>85 \%)$ at the single-seed level [9]. Genetic study of the trait in T2100 seeds indicated that the trait was controlled by recessive alleles at a single locus Tph 2 in such a way that Tph2Tph 2 and Tph 2 tph 2 genotypes produced low levels of gamma-tocopherol $(<5 \%)$ and tph 2 tph 2 genotypes showed high levels of gamma-tocopherol $(>85 \%)$, with no intermediate levels being observed [12]. On the contrary, IAST-1 derived from a mutagenesis program in which an $M_{2}$ seed with intermediate gamma-tocopherol content (19.2\%) was identified. The trait showed continuous variation for gamma-tocopherol levels (zero to 84.5\%) in the $\mathrm{M}_{3}$ generation, which allowed selection of genotypes with stable high gamma-tocopherol content in the $\mathrm{M}_{4}$ generation [11]. A comparative genetic study between both lines concluded that they shared the same allele at Tph2, as no transgressive segregation was observed in the $F_{1}$ and $F_{2}$ generations from crosses between them [13]. However, the evaluation of several $F_{2}$ populations from crosses of IAST-1 with T2100 and the conventional sunflower line HA89 showed that intermediate gamma-tocopherol values (5 to $85 \%$ ) occurred in some $F_{2}$ populations, whereas other $\mathrm{F}_{2}$ populations showed uniformly high gammatocopherol content (IAST-1 x T2100) or segregated into well-defined low and high gamma-tocopherol classes (IAST-1 x HA89) [17]. The results of this research suggest that intermediate gamma-tocopherol levels are produced by the effect of modifying genes on $t p h 2^{a}$ alleles from IAST-1. We hypothesize that modifying genes were present in the mutagenized population from which IAST1 was developed, where selection against negative alleles at modifying genes led to the isolation of IAST-1, and also in other lines such as HA89 and T2100. Modifying genes have no phenotypic effect in absence of alteration in the major gene [23]. In this research, it was also found that modifying genes have no phenotypic effect on the mutated allele at T2100 (tph2). Accordingly, they are expected to be segregating in HA89 and T2100 lines, which produced different segregation patterns in crosses with IAST-1 [17]. The occurrence of different segregation patterns depending on allelic configuration of modifying genes has been previously reported in sunflower for high oleic acid content $[24,25]$ and broomrape resistance [19].

Full length genomic gamma-TMT sequences corresponding to the Tph2 locus on LG 8 have been obtained in this study for both IAST-1 and nmsT2100. Both lines have two gamma-TMT paralogs. Paralog 1 is identical between the two lines and also to the gamma-TMT haplotype 4 from Hass et al. [14], which included sequences from both high and low gamma-tocopherol lines. Paralog 2 from IAST-1 is identical to gamma-TMT haplotype 5 from Hass et al. [14], which also included sequences from both high and low gamma-tocopherol lines, while paralog 2 from nmsT2100 carries slight SNP differences. Consequently, we have not found any sequence difference in the gamma-TMT gene from IAST-1 that would explain why the $t p h 2^{a}$ allele present in this line is unstable and affected by modifying genes. Previous research did not identify sequence differences in the gamma-TMT gene related to the high gammatocopherol phenotype [14]. The authors found identical gamma-TMT paralog 1 and 2 sequences in both high and low gamma-tocopherol lines and reported that mutation leading to the high gamma-tocopherol trait in the sunflower material derived from the high gamma-tocopherol line LG-17 [6] may disrupt regulatory sequences of the gamma-TMT gene. Relating the nmsT2100 line, the slight sequence differences in gamma-TMT paralog 2 were changes to those nucleotides also present in paralog 1 sequences from IAST-1 and nmsT2100 (2 SNPs in the 5' UTR region and in intron 1 ) or found in a repetitive sequence within an intron (3 SNPs in intron 4) and were not likely to be involved in determining a more stable gammaTMT allele.

Modifying genes affecting important traits for sunflower breeding such as high oleic acid content [24,25] and broomrape resistance [19] have been reported. The discovery of modifying genes affecting high gammatocopherol content confirms that the occurrence of modifying genes is not a rare phenomenon in sunflower genetics. Thus far there was no indication on the nature and mode of action of modifying genes. The results of the present research suggest that Tph2 modifying genes are in most cases additional gamma-TMT loci duplicated in the sunflower genome. This was confirmed for modifying genes at LG 1 and 16, whereas there is also indication that the modifying gene at LG 14 might be an additional copy of a gamma-TMT. Duplicated gammaTMT loci have also been found in safflower [26] and rapeseed [27]. In fact, the two gamma-TMT loci from safflower (one of them co-segregating with the safflower Tph2 gene determining high gamma-tocopherol content in this crop) were identified by using the F9/R24 primer combination from the sunflower gamma-TMT [26], the same one used in this study to map different gammaTMT loci. These results suggested that the F9 and R24 primer sequences are within a highly conserved region of the gamma-TMT gene. In sunflower, Hass et al. [14] mapped, in addition to gamma-TMT-Tph2 on LG 8, another gamma-TMT locus on LG 16, although the authors considered this locus a randomly amplified polymorphic DNA (RAPD). In this study, the locus on 
LG 16 (gamma-TMT locus $b$ ) showed significant homology to the sunflower gamma-TMT gene and underlay a gamma-tocopherol QTL. Our results indicated that interaction between duplicated gamma-TMT loci revert the high gamma-tocopherol phenotype to intermediate-low gamma-tocopherol values. This effect has also been reported for modifying genes affecting the high oleic acid phenotype in sunflower, where modifying genes suppressed the effect of the Ol-FAD2-1 allele that is essential for accumulating high oleic acid content $[18,28]$. Also, epistatically interacting duplicated MPBQ/MSBQ-MT genes that modify beta-tocopherol levels have been reported in sunflower [20]. It is well known that duplicate gene pairs can form negative epistasis due to their overlapping functions [29] and that suppression of a mutant phenotype can be altered by gene dosage [30]. However, additional biochemical, functional, and sequence analyses are required to determine the mode of action of duplicated gamma-TMT loci in sunflower.

From a breeding perspective, the existence of several modifying genes influencing high gamma-tocopherol content should not be a constraint for the development of cultivars with seeds rich in gamma-tocopherol, as this research revealed that modifying genes do not alter the phenotypic expression of the mutated allele tph 2 present at nmsT2100. On the other hand, the influence of modifying genes on expressivity of mutated $t p h 2^{a}$ alleles opens up the possibility of selection for balanced levels of alpha- and gamma-tocopherol content, provided that stable combinations of modifying genes can be obtained. The development of mid oleic acid hybrids of sunflower was based on genetic stocks carrying modifying genes that limit the expression of high oleic acid content produced by mutated alleles at $\mathrm{Ol}$ locus $(>80 \%)$ to the midrange level (55-75\%) [31]. In this sense, determination of allelic diversity at gamma-TMT loci underlying gamma-tocopherol modifying genes present in hybrid parental lines might be a useful tool to predict the presence of adequate allelic combinations giving rise to the desired levels of gamma-tocopherol content. Further studies should elucidate whether the other two gammatocopherol sources developed thus far, IAST-540 and LG-17, possess stable or unstable alleles at Tph2. Demurin et al. [6] reported differences in the expressivity of the recessive homozygotes of the $T p h 2$ gene from LG-17 in different genetic backgrounds, which might be indicative of allelic instability.

\section{Conclusions}

The results of this research suggest that high gammatocopherol in sunflower lines IAST-1 and nmsT2100 is determined by different mutated alleles at the gammaTMT Tph2 locus on LG 8. The allele at nmsT2100 is not affected by modifying genes, whereas the allele at IAST-1 is unstable and affected by modifying genes. Putative modifying genes have been located at LG 1, 9, 14 and 16, being the effect of those on LG 1,14 and 16 highly significant in their epistatic interaction with the Tph2 locus on LG 8. Finally, our results suggest that modifying genes correspond to gamma-TMT loci duplicated in the sunflower genome. Phenotypic effects of modifying genes altering the expression of important genes in crop plants are known [23], but in most cases the genetic basis for modification remains unclear. In this study, we shed light into the mode of action and nature of modifier genes in sunflower, and suggest the relevance of duplicated loci affecting the expression of seed quality specific mutations.

\section{Methods}

Plant material, phenotypic analyses and DNA extraction The study included the sunflower lines nmsT2100 and IAST-1, both with high gamma-tocopherol content (>85\%). nmsT2100 is a NMS line developed by introgressing monogenic recessive NMS from line P21 [32] into high gamma-tocopherol line T2100 [10]. IAST-1 was isolated in the course of a chemical mutagenesis program on seeds of an accession of 'Peredovik' [11]. Twenty-four half seeds of nmsT2100 and IAST-1 were nondestructively analyzed for tocopherol profile as described below, germinated and planted in pots under open air conditions in the spring of 2005. NMS plants of nmsT2100 were pollinated with pollen of IAST-1 plants. Half seeds of the parents as well as $F_{1}$ half seeds were analysed for tocopherol profile. $F_{1}$ and parent half seeds were sown in March 2006 and the corresponding plants were grown in pots under open air conditions. $F_{1}$ plants were bagged before flowering to obtain the $F_{2}$ generation. $F_{2}$ seeds from several $F_{1}$ plants were analysed for seed tocopherol profile. In most cases, $\mathrm{F}_{2}$ seeds had uniformly high gamma-tocopherol content (>90\%). However, some $F_{1}$ plants showed segregation for gammatocopherol content at the $F_{2}$ seed level, which indicated the expression of modifier genes. One population of $192 \mathrm{~F}_{2}$ seeds from a single $\mathrm{F}_{1}$ plant that showed large segregation for gamma-tocopherol content was selected for the molecular analyses. $F_{2}$ half seeds were germinated and the corresponding plants were grown in pots under open air conditions in 2007. $F_{2}$ plants were bagged before flowering to produce the $F_{3}$ seed. Five fully expanded leaves from each $\mathrm{F}_{2}$ plant ( $135 \mathrm{~F}_{2}$ plants) were cut, frozen at $-80^{\circ} \mathrm{C}$, lyophilised and ground to a fine powder in a laboratory mill. DNA was isolated from ground leaf tissue from each $F_{2}$ plant and from five plants of nmsT2100 and IAST-1 parental lines as described in Berry et al. [33]. Twenty four $F_{3}$ seeds from 
each $\mathrm{F}_{2}$ plant that produced sufficient amount of seeds were analysed for tocopherol profile.

Two additional $F_{2}$ populations were also used in this study with the objective of mapping tocopherol biosynthesis loci. One population, described in García-Moreno et al. [13], derived from a cross between CAS-12, with wild-type tocopherol profile mainly made up of alphatocopherol, and IAST-540, with high gamma-tocopherol content. The other population derived from a cross between lines IAST-413 and HA-89, both with wild-type tocopherol profile mainly made up of alpha-tocopherol, though IAST-413 is characterized by increased total tocopherol content [34].

The analysis of tocopherol profile was made for all analyzed generations and populations in half seeds. The half-seed technique is a common technique used in sunflower breeding consisting in cutting a small seed piece from the seed part distal to the embryo, which is used for nondestructive analysis of seed quality traits, as the remaining seed containing the embryo can be germinated after the corresponding analysis [35]. Individual half seeds were analysed for tocopherol profile following the method of Goffman et al. [36]. Half seeds were placed into $10-\mathrm{ml}$ tubes with $2 \mathrm{ml}$ iso-octane. The half seeds were then crushed with a stainless steel rod as fine as possible. The samples were stirred and extracted overnight at room temperature in darkness (extraction time about $16 \mathrm{~h}$ ). After extraction, the samples were stirred again, centrifuged, and filtered. Twenty-five $\mu \mathrm{l}$ of the extract were analysed by HPLC using a fluorescence detector at $295 \mathrm{~nm}$ excitation and $330 \mathrm{~nm}$ emission and iso-octane/tert-butylmethylether (94:6) as eluent at an isocratic flow rate of $1 \mathrm{ml} \mathrm{min}{ }^{-1}$. Chromatographic separation of the tocopherols was performed on a LiChrospher 100 diol column (250 mm x $2 \mathrm{~mm}$ I.D.) with $5-\mu \mathrm{m}$ spherical particles, connected to a silica guard column (LiChrospher Si 60, $5 \mathrm{~mm} \times 4 \mathrm{~mm}$ I.D.). The peak areas of the individual tocopherols were corrected according to their previously calculated response factors: alpha-tocopherol $=1.0$; beta-tocopherol $=1.80$; gammatocopherol $=1.85$; delta-tocopherol $=2.30$.

\section{Map construction and molecular analysis}

A complete linkage map for the nmsT2100 x IAST-1 population was constructed to scan the genome for modifier genes affecting the expression of the Tph2 gene. For this, the parental lines IAST-1 and nmsT2100 were initially screened for polymorphisms in two replicate samples together with four $\mathrm{F}_{2}$ individuals, using a genome-wide framework of 95 sunflower SSRs [37]. A preliminary genetic linkage map from this population was constructed. A set of INDEL markers [38], identified by ZVG prefixes, and an additional set of SSR markers mapped by Tang et al. [39] and Yu et al. [38], identified by ORS and CRT prefixes, were additionally screened for polymorphisms between these parental lines to complete the linkage map. INDEL markers for the tocopherol biosynthesis genes gamma-TMT, MPBQ/ MSBQ-MT and tocopherol cyclase described by Hass et al. [14] and Tang et al. [20] were also screened for polymorphisms between nmsT2100 and IAST-1. PCRs for SSRs analyses were performed as described by PérezVich et al. [40]. INDEL analyses were carried out following $\mathrm{Yu}$ et al. [38] and Hass et al. [14]. SSR and INDEL amplification products were separated on $3 \%(\mathrm{w} / \mathrm{v})$ Metaphor $^{\circledR}$ (BMA, Rockland, ME, USA) and 1.5\% agarose gels, respectively, in $1 \mathrm{x}$ TBE buffer with ethidium bromide incorporated in the gel. SSR and INDEL markers revealing polymorphisms were then genotyped in the nmsT2100 $\mathrm{x}$ IAST- $1 \mathrm{~F}_{2}$ population, following the protocols mentioned above.

Chi-square statistics were computed on each genotyped locus to detect deviations from the expected Mendelian ratios for codominant (1:2:1) or dominant (3:1) markers. The nmsT2100 x IAST-1 linkage map was constructed using the software MAPMAKER/EXP version 3.0b (Whitehead Institute, Cambridge, MA, USA) [41]. Two-point analysis was used to identify linkage groups (LGs) at a LOD score of 3 and a maximum recombination frequency of 0.40 . Three-point and multi-point analyses were used to determine the order and interval distances between the markers at each LG. The Haldane mapping function was used to compute the map distances in centiMorgans (cM) from the recombination fractions. Multiple loci detected by a single maker were coded with the marker name plus the suffix a, b, c, or d to indicate each duplicate locus. Linkage group maps were drawn using the MapChart software [42].

Genetic analysis of modifier genes was performed in several stages. In the first stage, the significance of each marker's association with the phenotypic trait [gammatocopherol content at the $\mathrm{F}_{2}$ seed and $\mathrm{F}_{2}$ plant (average value of $24 F_{3}$ seeds per $F_{2}$ plant) generations] was determined by one-way analysis of variance (ANOVA) using the statistical package SPSS Statistics v. 19, with marker genotypes being classes. In this analysis, we identified an unexpected macromutation on LG 8 at the Tph2 locus. The effects of the macromutations, if ignored, could dramatically reduce the power for identifying other genes or QTL affecting the studied trait.

In a second stage, composite interval mapping (CIM) $[43,44]$ was used to scan the genome for QTL affecting gamma-tocopherol content, in order to strengthen and corroborate the results of the analyses of variance, evaluate the existence of additional QTL, and estimate the interaction and global effect of all the detected QTL. Computations were carried out using the software PLABQTL Version 1.1 [45]. The phenotypic data 
consisted on gamma-tocopherol content in the $F_{2}$ seed and $F_{2}$ plant generations. Additional analyses were carried out by using other parameters calculated from the $\mathrm{F}_{3}$ seed data such as the minimum, maximum and the standard deviation of gamma-tocopherol content in $F_{3}$ seeds per $F_{2}$ plant, and the number of $F_{3}$ seeds within each $F_{2}$ plant with less than $90 \%$ of gamma-tocopherol content. Since these analyses gave similar results to those obtained with the mean gamma-tocopherol value per $F_{3}$ family, only results based on the $F_{3}$ mean value are shown. Analyses were made initially with the "first" statement to check the database for errors and outliers. Next, simple interval mapping (SIM) was carried out for an initial scan and detection of QTL with main effects. Finally, CIM was performed with markers closest to the main QTL as cofactors. Genome-wide threshold values $(\alpha=0.05)$ for declaring the presence of QTL were estimated from 1000 permutations of each phenotypic trait [46]. The thresholds of the LOD score (and their 0.95 confidence intervals) were $2.57(2.48-2.70)$ and $2.65(2.58-2.76)$ for gammatocopherol content in the $\mathrm{F}_{2}$ and the $\mathrm{F}_{3}$ generations, respectively. Estimates of QTL positions were obtained at the point where the LOD score reaches its maximum in the region under consideration. One-LOD support limits for the position of each QTL were also calculated [47]. The proportion of phenotypic variance explained by each individual QTL was calculated as the square of the partial correlation coefficient $\left(R^{2}\right)$. Estimates of the additive $\left(\mathrm{a}_{\mathrm{i}}\right)$ and dominance $\left(\mathrm{d}_{\mathrm{i}}\right)$ effects, as defined by Falconer [48], for the ith putative QTL, the total LOD score, as well as the total proportion of the phenotypic variance explained by all QTL, were obtained by fitting a multiple regression model including all putative QTL for the respective trait simultaneously [47]. The occurrence of QTL x QTL interactions was tested by adding digenic epistatic effects to the model.

QTL software such as PLABQTL estimate epistatic interactions among previously identified QTL. Since modifying genes are defined as genes having no known effect except to intensify or diminish the expression of a major gene [23], their effect as individual loci and subsequently their interaction with major loci may be undetectable with this type of analyses. In consequence, two-way interactions between the Tph 2 major locus and all the marker loci genotyped in this study were also tested. Twoway interactions were analyzed at a significance threshold of $P \leq 0.05$ by analysis of variance using the general linear model (GLM) of SPSS Statistics v. 19. Statistical significance of differences for gamma-tocopherol content in different genotypes combining two marker loci were also computed using Duncan's multiple range test. The significant epistatic interaction terms were combined with those of the previously identified QTL in multiple locus models using the "seq" statement of PLABQTL.

\section{Sequence analysis gamma-TMT loci}

In the course of the genetic analyses of the nmsT2100 $\mathrm{x}$ IAST-1 population, we identified different gamma-TMT loci amplified with INDEL marker gamma-TMT-F9/R24 associated to gamma-tocopherol QTL. In order to confirm their nature, these loci were sequenced as follows. F9/R24 INDEL fragments amplified from the IAST-1 and nmsT2100 parental lines were separated on a 1.5\% agarose gel, excised and purified by means of the QIAquick gel extraction kit (Qiagen GmbH, Hilden, Germany). The purified fragments were ligated into the T/A vector (pCR2.1) and the recombinants were transformed to TOP10 Chemically Competent E. coli using the TOPO-TA cloning kit (Invitrogen, San Diego, CA, USA) as described by the manufacturer. Five recombinant bacterial colonies (white) per isolated band were picked from the plate containing ampicillin and X-gal as selective media and cultured overnight at $37^{\circ} \mathrm{C}$. Plasmids were extracted and purified using QIAprep Spin Miniprep Kit (Qiagen GmbH, Hilden, Germany). PCR with M13 forward and reverse vector primers and F9 and R24 primers, and restriction enzyme digestion was performed to confirm the presence and size of the insert. Sequencing in both forward and reverse orientations of the cloned fragments (two clones per locus) was performed at GATC Biotechnology (Konstanz, Germany) using the M13 forward and reverse sequencing primers. Sequence analysis was conducted with the aid of the software Vector NTI Advance 10.3.0 (Invitrogen, San Diego, CA, USA).

\section{Full-length sequence analysis of the IAST-1 and nmsT2100 gamma-TMT gene}

Full-length gamma-TMT genomic DNA sequences were isolated from the high gamma-tocopherol lines nmsT2100 and IAST-1 by long distance PCR using primers developed at the $5^{\prime}$ end (forward primer gamma-TMT-F1 from Hass et al. [14]) and the 3'end (reverse primer R92: TAATTCCTTGGGATGCCATT) of the sunflower gamma-TMT gene (GenBank accessions nos. DQ229828 to DQ229834). AccuPrime High Fidelity Taq DNA Polymerase (Invitrogen Life Technologies, Carlsbad, CA, USA) was used for PCR amplification in three individuals of nmsT2100 and five of IAST-1 as described by the manufacturer. The amplified products from each individual were separated on $1.5 \%$ agarose gels, showing in both lines two bands of a size higher than $4 \mathrm{~kb}$ that corresponded to the two gamma-TMT paralogs described by Hass et al. [14]. The upper (paralog 2) and the lower (paralog 1) bands were independently purified in each individual and cloned using the TOPO-TA cloning kit (Invitrogen Life Technologies, Carlsbad, CA, USA) as described above, with the exception that twenty recombinant bacterial colonies (white) per isolated band were picked from the plate. Restriction enzyme digestion was 


\begin{tabular}{ll}
$\begin{array}{l}\text { Table } \mathbf{5} \text { Sequencing primers designed each } \mathbf{5 0 0 - 1 0 0 0} \text { bp } \\
\text { into the gamma-TMT gene }\end{array}$ \\
\hline Primer & Sequence (5'-3') \\
\hline F90 & GGATGAATCGTTGTTATTG \\
F91 & GTCAATGGAGAGTGGAGAGC \\
F92 & AGGAAGAAAAATCTTGAATAA \\
F93 & ATCGCTTCATCATCATCATA \\
F94 & CACTAAATTGACATCCACAAC \\
F95 & GCCACTAATGATTGAAGGAT \\
R94 & ACCACAACGTAAAAATGTT \\
R95 & CCACTACGTAGCAATGAAGT \\
R96 & CCTTAGTTTGCAATTCAC \\
R97 & CCGAGTCAACTCACTAACAA \\
R98 & TCATTCACAAACTGCAGTAG
\end{tabular}

performed to confirm the presence of the insert and the restriction patterns characteristic for each gamma-TMT paralog. Sequencing in both forward and reverse orientations of the cloned fragments (a total of 10 clones for IAST-1 paralog 1, 13 clones for IAST-1 paralog 2, 5 clones for nmsT2100 paralog 1, and 6 clones for nmsT2100 para$\log 2$ ) was performed at GATC Biotechnology (Konstanz, Germany) using the universal M13 forward and reverse primers and internal primers (Gamma-TMT-F9, F27, F67, R10, R24, R35 and R78 from Hass et al. [14], and primers from Table 5) designed at 500 to $1000 \mathrm{bp}$ intervals from the sunflower gamma-TMT gene. Sequence analysis was conducted using Vector NTI Advance 10.3.0 (Invitrogen, San Diego, CA, USA). A consensus sequence for IAST-1 gamma-TMT paralog 1, IAST-1 gamma-TMT paralog 2, nmsT2100 gamma-TMT paralog 1, and nmsT2100 gamma-TMT paralog 2 was made from the analysis of 10, 13,5 , and 6 sequenced clones, respectively. Changes in the nucleotide sequence were only included in the consensus sequence when they were conserved among the different clones and individuals sequenced from each line.

\section{Additional file}

Additional file 1: Figure S1. Gamma-TMT sequence alignment.

Sunflower gamma-tocopherol methyltransferase genomic DNA sequence alignment for the high gamma-tocopherol lines IAST-1 and nmsT2100 as well as genbank sequences from gamma-TMT haplotypes 1 (DQ DQ229828), 2 (DQ DQ229829), 3 (DQ DQ229830), 4 (DQ DQ229831 and DQ DQ229832), and 5 (DQ DQ229833 and DQ DQ229834) and the CDNA gamma-TMT EF495161 sequence.

\footnotetext{
Abbreviations

Gamma-TMT: gamma-tocopherol methyltransferase; INDEL: insertiondeletion; LG: linkage group; MPBQ/MSBQ-MT: 2-methyl-6-phytyl-1,4benzoquinone/2-methyl-6-solanyl-1,4-benzoquinone methyltransferase; NMS: nuclear male sterile; SSR: simple sequence repeat.
}

\section{Authors' contributions}

MJG-M carried out genetic map construction and all other molecular analyses. JMF-M and LV selected the sunflower lines, crossed them, produced and studied the phenotypic data, and participated in the design of the study. BP-V conceived and designed the study and supervised molecular analyses and interpretation of results. All authors contributed to the manuscript preparation, and read and approved the final manuscript.

\section{Acknowledgements}

The research was funded by the Spanish Ministry of Science and Innovation and the European Union FEDER (research project AGL2007-62834) and Dow Agrosciences LLC. We acknowledge support of the publication fee by the CSIC Open Access Publication Support Initiative through its Unit of Information Resources for Research (URICI).

Received: 7 September 2011 Accepted: 1 May 2012

Published: 18 May 2012

\section{References}

1. Eitenmiller R, Lee J: Vitamin E. Food Chemistry, Composition, and Analysis, New York. New York: Marcel Dekker; 2004.

2. Hunter SC, Cahoon EB: Enhancing vitamin E in oilseeds: Unravelling tocopherol and tocotrienol biosynthesis. Lipids 2007, 42:97-108.

3. Chow CK: In Vitamin E. Edited by Stipanuk MH. Philadelphia: Saunders; 2000:584-598.

4. Kamal-Eldin A, Appelqvist L-Å: The chemistry and antioxidant properties of tocopherols and tocotrienols. Lipids 1996, 31:671-701.

5. Seppanen CM, Song Q, Csallany AS: The antioxidant functions of tocopherol and tocotrienol homologues in oils, fats, and food systems. Journal of the American Oil Chemists' Society 2010, 87:469-481.

6. Demurin Y, Skoric D, Karlovic D: Genetic variability of tocopherol composition in sunflower seeds as a basis of breeding for improved oil quality. Plant Breeding 1996, 115:33-36.

7. Fuster MD, Lampi AM, Hopia A, Kamal-Eldin A: Effects of alpha- and gamma-tocopherols on the autoxidation of purified sunflower triacylglycerols. Lipids 1998, 33:715-722

8. Yanishlieva NV, Kamal-Eldin A, Marinova EM, Toneva AG: Kinetics of antioxidant action of alpha- and gamma-tocopherols in sunflower and soybean triacylglycerols. European Journal of Lipid Science and Technology 2002, 104:262-270.

9. Marmesat S, Velasco L, Ruiz-Méndez MV, Fernández-Martínez JM, Dobarganes C: Thermostability of genetically modified sunflower oils differing in fatty acid and tocopherol compositions. European Journal of Lipid Science and Technology 2008, 110:776-782.

10. Velasco L, Dominguez J, Fernández-Martínez JM: Registration of T589 and T2100 sunflower germplasms with modified tocopherols. Crop Sci 2004, 44:362-363.

11. Velasco L, Pérez-Vich B, Fernández-Martínez JM: Novel variation for tocopherol profile in a sunflower created by mutagenesis and recombination. Plant Breeding 2004, 123:490-492.

12. Velasco L, Fernández-Martínez JM: Identification and genetic characterization of new sources of beta- and gamma-tocopherol in sunflower germplasm. Helia 2003, 26:17-24.

13. García-Moreno MJ, Vera-Ruiz EM, Fernández-Martínez JM, Velasco L, PérezVich B: Genetic and molecular analysis of high gamma-tocopherol content in sunflower. Crop Sci 2006, 46:2015-2021.

14. Hass CG, Tang S, Leonard S, Traber M, Miller JF, Knapp SJ: Three non-allelic epistatically interacting methyltransferase mutations produce novel tocopherol (vitamin E) profiles in sunflower. Theor Appl Genet 2006, 113:767-782.

15. Bergmüller E, Porfirova S, Dörmann P: Characterization of an Arabidopsis mutant deficient in $\mathrm{y}$-tocopherol methyltransferase. Plant Mol Biol 2003, 52:1181-1190

16. Demurin Y, Efimenko SG, Peretyagina TM: Genetic identification of tocopherol mutations in sunflower. Helia 2004, 27:113-116.

17. García-Moreno MJ, Fernández-Martínez JM, Pérez-Vich B, Velasco L: In A modifying gene affecting gamma-tocopherol content in sunflower, Proceedings of the Seventeenth International Sunflower Conference: 8-12 June 2008; Córdoba, Spain. Edited by Velasco L. Paris: International Sunflower Association; 2008:601-604. 
18. Lacombe $S$, Kaan F, Léger $L$, Bervillé $A$ : An oleate desaturase and a suppressor loci direct high oleic acid content of sunflower (Helianthus annuus L.) oil in the Pervenets mutant. Comptes Rendus de l'Academie des Sciences, Series III, Sciences de la Vie 2001, 324:839-845.

19. Velasco L, Pérez-Vich B, Jan CC, Fernández-Martínez JM: Inheritance of resistance to broomrape (Orobanche cumana Wallr.) race $F$ in a sunflower line derived from wild sunflower species. Plant Breeding 2007, 126:67-71.

20. Tang S, Hass C, Knapp S: Ty3/gypsy-like retrotransposon knockout of a 2methyl-6-phytyl-1,4-benzoquinone methyltransferase is non-lethal, uncovers a cryptic paralogous mutation, and produces novel tocopherol (vitamin E) profiles in sunflower. Theor Appl Genet 2006, 113:783-799.

21. Pérez-Vich B, Berry ST, Velasco L, Fernández-Martínez JM, Gandhi S, Freeman C, Heesacker A, Knapp SJ, Leon AJ: Molecular mapping of nuclear male sterility genes in sunflower. Crop Sci 2005, 54:1851-1857.

22. Del Moral L, Fernández-Martínez JM, Pérez-Vich B, Velasco L: Expression of modified tocopherol content and profile in sunflower tissues. J Sci Food Agric. 2012, 92:351-357.

23. Briggs FD, Knowles PF: Introduction to Plant Breeding. New York: Reinhold Publishing Corporation; 1967.

24. Urie AL: Inheritance of high oleic acid in sunflower. Crop Sci 1985, 25:986-989.

25. Velasco L, Pérez-Vich B, Fernández-Martínez JM: Inheritance of oleic acid content under controlled environment. In Proceedings of the Fifteenth International Sunflower Conference: 12-15: Toulouse, France. Paris: International Sunflower Association June 2000, 2000:A31-A36.

26. García-Moreno MJ, Fernández-Martínez JM, Velasco L, Pérez-Vich B: Molecular tagging and candidate gene analysis of the high gammatocopherol trait in safflower (Carthamus tinctorius L.). Mol Breed 2011, 28:367-379.

27. Endrigkeit J, Wang X, Cai D, Zhang C, Long Y, Meng J, Jung C: Genetic mapping, cloning, and functional characterization of the BnaX.VTE4 gene encoding a gamma-tocopherol methyltransferase from oilseed rape. Theor Appl Genet 2009, 119:567-575.

28. Fernández-Martínez JM, Jiménez A, Domínguez J, García JM, Garcés R, Mancha M: Genetic analysis of the high oleic content in cultivated sunflower (Helianthus annuus L.). Euphytica 1989, 41:39-51.

29. Jiang $H, G u Z$ : Growth of novel epistatic interactions by gene duplication. Genome Biology and Evolution 2011, 3:295-301.

30. Prelich G: Suppresion mechanisms. Trends in Genetics 1999, 15:261-266.

31. Miller JF, Vick BA: Registration of four mid-range oleic acid sunflower genetic stocks. Crop Sci 2002, 42:994.

32. Jan CC: Inheritance and allelism of mitomycin C- and streptomycininduced recessive genes for male sterility in cultivated sunflower. Crop Sci 1992, 32:317-320.

33. Berry ST, Leon AJ, Hanfrey CC, Challis P, Burkholz A, Barnes SR, Rufener GK, Lee M, Caligari PDS: Molecular marker analysis of Helianthus annuus L. 2. Construction of an RFLP linkage map for cultivated sunflower. Theor Appl Genet 1995, 91:195-199.

34. Del Moral L, Pérez-Vich B, Fernández-Martínez JM, Velasco L: Inheritance of increased seed tocopherol content in sunflower line IAST-413. Plant Breeding. 2011, 130:540-543.

35. Fernández-Martínez JM, Perez-Vich B, Velasco L: In Sunflower, Oil Crops. Edited by Vollmann J, Rajcan I. New York: Springer; 2009:155-232.

36. Goffman FD, Velasco L, Thies W: Quantitative determination of tocopherols in single seeds of rapeseed (Brassica napus L.). Fett-Lipid 1999, 101:142-145.

37. Tang S, Kishore VK, Knapp SJ: PCR-multiplexes for a genome-wide framework of simple sequence repeat marker loci in cultivated sunflower. Theor Appl Genet 2003, 107:6-19.

38. Yu JK, Tang S, Slabaugh MB, Heesacker A, Cole G, Herring M, Soper J, Han F, Chu W-C, Webb DM, Thompson L, Edwards KJ, Berry S, Leon AJ, Grondona M, Olungu C, Maes N, Knapp SJ: Towards a saturated molecular genetic linkage map for cultivated sunflower. Crop Sci 2003, 43:367-387.

39. Tang S, Yu JK, Slabaugh MB, Shintani DK, Knapp SJ: Simple sequence repeat map of the sunflower genome. Theor Appl Genet 2002, 105:1124-1136.

40. Pérez-Vich B, Akhtouch B, Knapp SJ, Leon AJ, Velasco L, Fernández-Martínez JM, Berry ST: Quantitative trait loci for broomrape (Orobanche cumana Wallr.) resistance in sunflower. Theor Appl Genet 2004, 109:92-102.
41. Lander ES, Green P, Abrahamson J, Barlow A, Daly MJ, Lincoln SE, Newburg L: MAPMAKER: An interactive computer package for constructing primary genetic linkage maps of experimental and natural populations. Genomics 1987, 1:174-181.

42. Voorrips RE: MapChart: Software for the graphical presentation of linkage maps and QTL. J Hered 2002, 93:77-78.

43. Jansen RC, Stam P: High resolution of quantitative traits into multiple loci via interval mapping. Genetics 1994, 136:1447-1455.

44. Zeng ZB: Precision mapping of quantitative trait loci. Genetics 1994, 136:1457-1468.

45. Utz HF, Melchinger AE: PLABQTL: A program for composite interval mapping of QTL. Journal of Quantitative Trait Loci 1996, 2:1-5.

46. Doerge RW, Churchill GA: Permutation tests for multiple loci affecting a quantitative character. Genetics 1996, 142:285-294.

47. Bohn M, Khairallah MM, González-de-León D, Hoisington DA, Utz HF Deutsch JA, Jewell DC, Mihm JA, Melchinger AE: QTL mapping in tropical maize: I. Genomic regions affecting leaf feeding resistance to sugarcane borer and other traits. Crop Sci 1996, 36:1352-1361.

48. Falconer DS: Introduction to quantitative genetics. 3rd edition. London: Longman; 1989

doi:10.1186/1471-2229-12-71

Cite this article as: García-Moreno et al:: Genetic basis of unstable expression of high gamma-tocopherol content in sunflower seeds. BMC Plant Biology 2012 12:71.

\section{Submit your next manuscript to BioMed Central and take full advantage of:}

- Convenient online submission

- Thorough peer review

- No space constraints or color figure charges

- Immediate publication on acceptance

- Inclusion in PubMed, CAS, Scopus and Google Scholar

- Research which is freely available for redistribution 